\title{
Backlash Compensation for Plants With Saturating Actuators
}

DOI:

$10.1177 / 0959651817692471$

\section{Document Version}

Accepted author manuscript

Link to publication record in Manchester Research Explorer

\section{Citation for published version (APA):}

Rodríguez-Liñán, M. D. C., \& Heath, W. (2017). Backlash Compensation for Plants With Saturating Actuators. Institution of Mechanical Engineers. Proceedings. Part I: Journal of Systems and Control Engineering. https://doi.org/10.1177/0959651817692471

\section{Published in:}

Institution of Mechanical Engineers. Proceedings. Part I: Journal of Systems and Control Engineering

\section{Citing this paper}

Please note that where the full-text provided on Manchester Research Explorer is the Author Accepted Manuscript or Proof version this may differ from the final Published version. If citing, it is advised that you check and use the publisher's definitive version.

\section{General rights}

Copyright and moral rights for the publications made accessible in the Research Explorer are retained by the authors and/or other copyright owners and it is a condition of accessing publications that users recognise and abide by the legal requirements associated with these rights.

\section{Takedown policy}

If you believe that this document breaches copyright please refer to the University of Manchester's Takedown Procedures [http://man.ac.uk/04Y6Bo] or contact uml.scholarlycommunications@manchester.ac.uk providing relevant details, so we can investigate your claim.

\section{OPEN ACCESS}




\section{Backlash Compensation for Plants}

\section{With Saturating Actuators}

\section{Ma. del Carmen Rodríguez-Liñán ${ }^{1}$ and William Heath ${ }^{2}$}

Journal Title

$X X(X): 1-17$

(C)The Author(s) 0000

Reprints and permission:

sagepub.co.uk/journalsPermissions.nav

DOI: $10.1177 /$ ToBeAssigned

www.sagepub.com/

SAGE

\begin{abstract}
A compensation scheme is proposed for plants subject to both saturation and backlash in series at the actuator. It is shown that the series connection of a backlash inverse, saturation and the backlash itself is equivalent to a saturation function at a different level to the original saturation; we call this the 'saturation equivalence'. Therefore it is proposed to include such a backlash inverse in the compensator. This is shown to be optimal in a sense defined in the paper. It is then straightforward to devise a compensator scheme based on traditional anti-windup. This is illustrated in both simulation and on a laboratory scale rig with severe backlash. A correction for the chattering observed in the control signal due to the discontinuous nature of the nonlinearity inverse is also presented.
\end{abstract}

\title{
Keywords
}

Control system design, non-linear control, backlash compensation, backlash inverse, anti-windup, mechanical control systems

\section{Introduction}

Physical plants are commonly affected by nonlinearities such as backlash, while actuator saturation is always present. The occurrence of such nonlinearities in a given plant usually causes a degradation of the closed-loop system's performance; without appropriate compensation mechanisms (such as inversion or anti-windup) such

\footnotetext{
${ }^{1}$ Cátedra CONACyT at Instituto Tecnológico de Ensenada.

2 School of Electrical and Electronic Engineering, University of Manchester.
}

Corresponding author:

Ma. del Carmen Rodríguez-Liñán, Instituto Tecnológico de Ensenada, 
degradation can be catastrophic. In this paper a novel compensation scheme is proposed for plants that suffer from both saturation and a backlash nonlinearity in the actuator, as in Figure 1.

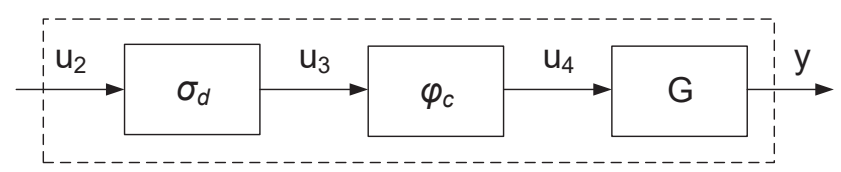

Figure 1. Studied plant dynamics, represented by three blocks in series. The block $\sigma_{d}$ represents actuator saturation and $\varphi_{c}$ represents the backlash nonlinearity. The block $G$ represents the remaining dynamics, assumed to be linear. Here $u_{2}$ represents the signal delivered to the plant, while $u_{3}$ and $u_{4}$ are internal states.

\subsection{Compensation strategies for backlash}

Backlash is a dynamic nonlinearity. It can produce delays in the output with respect to the input and loss of information. Typical examples of backlash are mechanical gears, robotic arms, servo-lenses etc, as discussed by Tao and Kokotović [1] whose treatment has greatly influenced the control literature for such nonlinearities when they occur in actuators. They propose the construction of an adaptive inverse, such that the effects of backlash are cancelled. Then, a control structure is used to achieve the required performance.

Several applications of this idea can be found in the literature. Zhou et al. in [2] construct a backlash inverse together with a backstepping adaptive controller. Agrawal et al. [3] provide a different version of a backlash inverse and use it in a robotic arm. In [4] Selmic and Lewis use neural networks and backstepping to calculate and compensate for the backlash. Consideration of plants with either backlash or saturation can be found in [5] using PID fractional algorithms and describing function analysis. Nordin and Gutman in [6] provide a detailed survey on compensation strategies for backlash.

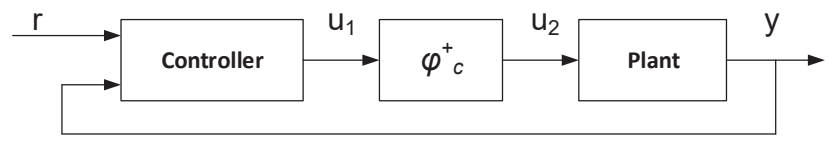

Figure 2. Compensation for plants with actuator backlash. As in Figure $1, u_{2}$ represents the signal delivered to the plant. The block $\varphi_{c}^{+}$represents a backlash right inverse. The controller is then designed for the linearised dynamics from $u_{1}$ to $y$.

\subsection{Saturation and anti-windup}

Physical systems are always bounded by actuator constraints; these could be limits on speed, voltage, torque, etc. If the signal commanded by the controller exceeds the limits of the actuator, then actuator saturation takes place and the efficiency of the controller will be reduced. The saturation characteristic can be described as a memoryless nonlinearity that maps small input signals linearly to the Boulevard Tecnológico 150, Ex-Ejido Chapultepec, 22780, Ensenada,

Baja California, Mexico.

Email: macaroli270@yahoo.com.mx 
output. When the input exceeds a limit value $\pm d$, the output remains constant and equal to the limit, referred to as the saturation level. When this happens, the action of the controller is restricted leading to loss of performance and possibly instability of the system.

A 'quick fix' to the saturation problem would be to enlarge the actuators so that the limits are never exceeded by the control action. However, this is usually an impractical and unrealistic solution [7]. More efficient is to design the controllers with the saturations in mind to exploit the capacity of the actuator in full. However, this does not come freely because it can give rise to the 'windup' problem. This refers to the damage to the controller's performance when the constraints are exceeded, [7],[8]. Strategies that are meant to solve the 'windup' problem are traditionally called 'anti-windup' compensators [7].

The basic structure of an anti-windup augmentation is shown in Figure 3, where $u_{0}$ is the output of the unconstrained controller and $u_{1}=\sigma_{d} u_{0}$. When the actuator is not saturated, the controller with anti-windup augmentation should yield the same response as the unconstrained controller. Conversely, when the saturation is active the output should approximate the unconstrained response in the best possible way. These two properties, called respectively small signal preservation and large signal recovery, are the basic properties any anti-windup augmentation must satisfy, [7],[8].

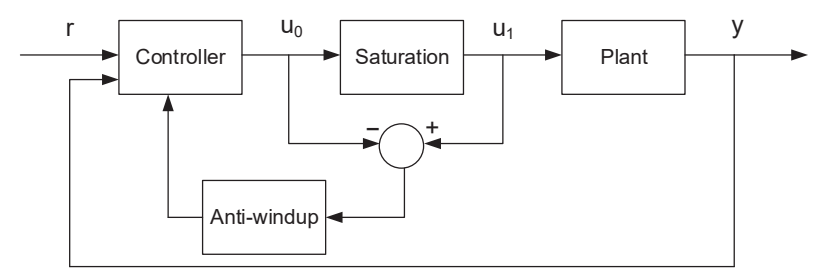

Figure 3. Basic anti-windup structure, $r$ represents the reference signal, $y$ is the output of the plant, $u_{1}$ is the output of the unconstrained controller, and $u_{1}=\operatorname{sat}\left(u_{0}\right)$ is the signal delivered to the plant.

Different anti-windup strategies, ranging from the very simple [9],[10] to the more sophisticated, [11],[7],[8], can be found in the literature.

\subsection{Compensation for combined backlash}

\section{and saturation}

Design recommendations for plants exhibiting both saturation and backlash are more rare. Zabiri and Samyudia in [12]-[13] propose using MPC (model predictive control) for a plant subject to backlash and saturation. They use constraints to characterize the backlash inverse and the saturation. These constraints are included in the MPC design requiring the online solution of a mixed-integer quadratic program. A stability analysis for control systems with such nonlinearities can be found by [14], [15] using Lyapunov functions and LMIs.

Utilizing generalized sector conditions, stability for systems with backlash and saturation is studied in [16]. Specifically, the focus is on systems with saturation in the 
input and backlash in the output. The authors obtain a Lyapunov function to derive local or global stability of the equilibrium. saturation. An MPC design based on the same results was proposed by the same authors in [19].

\section{Description of nonlinearities}

\subsection{Backlash operator}

This paper proposes a novel compensation strategy for plants that exhibit both backlash and saturation at the actuator. It is shown that the series interconnection of a backlash inverse, a saturation and the backlash itself results in a simple saturation, albeit with different bounds to the original saturation; this property is defined as "saturation equivalence". Furthermore the implementation of such a backlash inverse is shown to be optimal in a sense defined in the paper. The resulting nonlinearity can be compensated with a controller suitable for actuator saturation such as anti-windup. We illustrate the idea with simple PID antiwindup in both simulation and applied to a laboratory rig suffering from severe backlash.

The right inverse backlash is a discontinuous function everywhere. This means that chattering can appear in the control signal. This, however, is overcome using a spatial regularization technique similar to those discussed by Johansson et al. in[17].

Some preliminary results were presented by RodríguezLiñán and Heath in [18] where a similar control strategy was also proposed for plants with both deadzone and
Following [1] let $\varphi_{c}$ be the backlash operator mapping $u$ to $v$, characterized as

$$
\dot{v}(t)= \begin{cases}\dot{u}(t), & \text { if } \dot{u}(t)>0 \text { and } v(t)=u(t)-c \\ & \text { or } \dot{u}(t)<0 \text { and } v(t)=u(t)+c \\ 0, & \text { otherwise }\end{cases}
$$

with $c>0$, where $\dot{u}(t), \dot{v}(t)$ represent the time-derivatives of $u(t)$ and $v(t)$, respectively. Figure 4 illustrates (1).

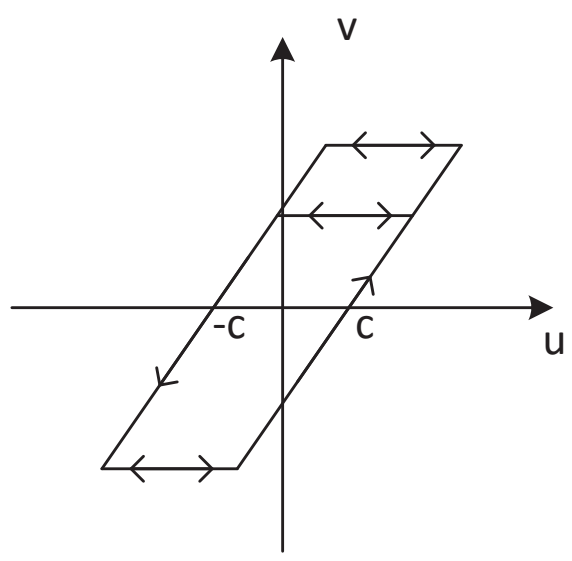

Figure 4. Backlash, $\varphi_{c}$.

\subsection{Inverse backlash}

Again following [1] we define the right inverse of an operator in the following sense. 
Definition 1. Let $\varphi$ and $\varphi^{+}$be operators such that

$$
v=\varphi^{+}(u) \text { and } w=\varphi(v) \text { for some } u, v \text { and } w .
$$

Then $\varphi^{+}$is the right inverse of $\varphi$ if

$$
w(t)=u(t) \text { for all } t \geq t_{0} \text { whenever } w\left(t_{0}\right)=u\left(t_{0}\right) .
$$

Following [1], let $\varphi_{c}^{+}$be the operator mapping $u$ to $v$, characterised as

$$
\dot{v}(t)= \begin{cases}\dot{u}(t), & \text { if } \dot{u}(t)>0 \text { and } v(t)=u(t)+c, \\ 0, & \text { or } \dot{u}(t)<0 \text { and } v(t)=u(t)-c, \\ g(t, t), & \text { if } \dot{u}(t)=0, \\ -g(t, t), & \text { if } \dot{u}(t)>0 \text { and } v(t)=u(t)-c,\end{cases}
$$

where

$$
g(\tau, t)=2 c \delta(\tau-t),
$$

with $\delta(t)$ as the Dirac $\delta$-function. Thus, $g(t, t)$ in equation (4) indicates that $g(\tau, t)$ is illustrated with $\tau=t$.

The operator $\varphi_{c}^{+}$is illustrated in Figure 5.

Lemma 1. [1]. The operator $\varphi_{c}^{+}$is the right inverse of $\varphi_{c}$.

\subsection{Saturation}

Let $\sigma_{d}$ be the operator mapping $u$ to $v$, characterised as

$$
v(t)=\operatorname{sat}_{d}(u(t))
$$

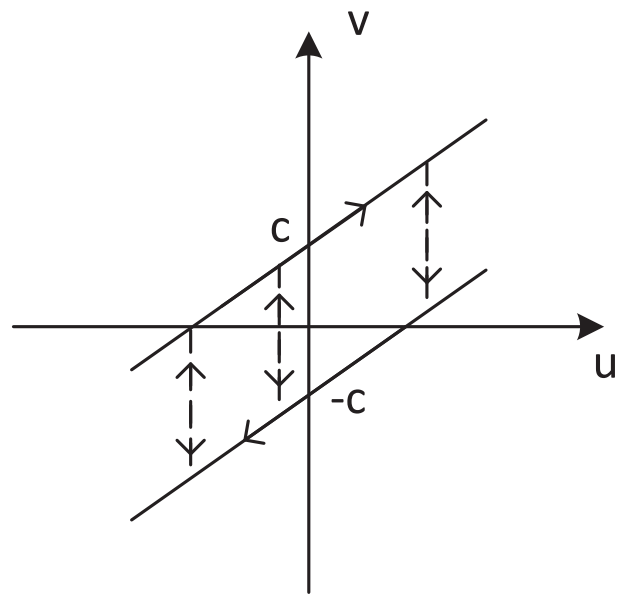

Figure 5. Right inverse of backlash, $\varphi_{c}^{+}$.

where

$$
\operatorname{sat}_{d}(u(t))= \begin{cases}d, & \text { if } u(t)>d, \\ u(t), & \text { if }|u(t)| \leq d, \\ -d, & \text { if } u(t)<-d,\end{cases}
$$

Figure 6. Saturation. 


\section{Proposed control structure}

\subsection{Saturation equivalence}

The main technical result of this paper is to show that the combination of the inverse nonlinearity with a saturation and the nonlinearity itself results in a saturation at a different level.

Theorem 1. Suppose for all continuous and differentiable signals $u$

$$
y=\varphi_{c}(w), w=\sigma_{d}(v) \text { and } v=\varphi_{c}^{+}(u), \text { with } d>c>0 .
$$

Then if

$$
y\left(t_{0}\right)=\operatorname{sat}_{d-c}\left(u\left(t_{0}\right)\right) \text { for some } t_{0},
$$

then

$$
y(t)=\operatorname{sat}_{d-c}(u(t)) \text { for all } t \geq t_{0}
$$

Proof. Three cases are considered.

Case 1. Suppose $|u(t)| \leq d-c$ for some interval $\left[t_{1}, t_{2}\right]$. Then $w(t)=v(t)$ for all $t \in\left[t_{1}, t_{2}\right]$. It follows that if $y\left(t_{1}\right)=u\left(t_{1}\right)$ then $y(t)=u(t)$ for all $t \in\left[t_{1}, t_{2}\right]$.

Case 2. Suppose $u(t) \geq d-c$ for some interval $\left[t_{1}, t_{2}\right]$. Then $v(t) \geq d-2 c$ and $d-2 c \leq w(t) \leq d$ for all $t \in\left[t_{1}, t_{2}\right]$. If $y\left(t_{1}\right)=d-c$ then $\dot{y}(t)=0$ for all $t \in\left[t_{1}, t_{2}\right]$ and hence $y(t)=d-c$ for all $t \in\left[t_{1}, t_{2}\right]$.

Case 3. Suppose $u(t) \leq-(d-c)$ for some interval $\left[t_{1}, t_{2}\right]$. Then similar to Case 2, $y(t)=-(d-c)$ for all $t \in$ $\left[t_{1}, t_{2}\right]$
To complete the proof the transitions between levels must be considered. Only transitions between when $|u(t)| \leq d-c$ and $u(t) \geq d-c$ (in both directions) will be considered. Conditions for transitions between when $|u(t)| \leq d-c$ and $u(t) \geq-(d-c)$ follow by symmetry. Since $u(t)$ is continuous, these are sufficient to show that $(9) \Rightarrow(10)$.

Transition 1. Suppose $|u(t)| \leq d-c$ for some interval $\left[t_{1}, t_{2}\right]$ and $u(t) \geq d-c$ for some interval $\left[t_{2}, t_{3}\right]$. If $y\left(t_{1}\right)=u\left(t_{1}\right)$ then $y(t)=u(t)$ for all $t \in\left[t_{1}, t_{2}\right]$; in particular $y\left(t_{2}\right)=d-c$. Hence $y(t)=d-c$ for all $t \in\left[t_{2}, t_{3}\right]$

Transition 2. Suppose $u(t) \geq d-c$ for some interval $\left[t_{1}, t_{2}\right]$ and $|u(t)| \leq d-c$ for some interval $\left[t_{2}, t_{3}\right]$. If $y\left(t_{1}\right)=$ $d-c$ then $y(t)=d-c$ for all $t \in\left[t_{1}, t_{2}\right]$; in particular $y\left(t_{2}\right)=u\left(t_{2}\right)$. Hence $y(t)=u(t)$ for all $t \in\left[t_{2}, t_{3}\right]$.

With some slight abuse of notation, it can be said that

$$
\varphi_{c} \sigma_{d} \varphi_{c}^{+}=\sigma_{d-c}
$$

Since

$$
\sigma_{d-c} \sigma_{d-c}=\sigma_{d-c}
$$

it follows immediately that one can say, with the same abuse of notation,

$$
\varphi_{c} \sigma_{d} \varphi_{c}^{+} \sigma_{d-c}=\sigma_{d-c}
$$




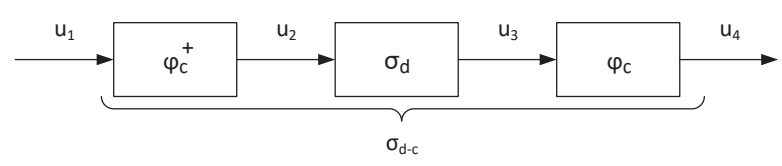

Figure 7. Series interconnection of the right inverse $\varphi_{c}^{+}$of the nonlinearity $\varphi_{c}$, a saturation and the nonlinear operator $\varphi_{c}$. This is equivalent to a saturation $\sigma_{d-c}$.

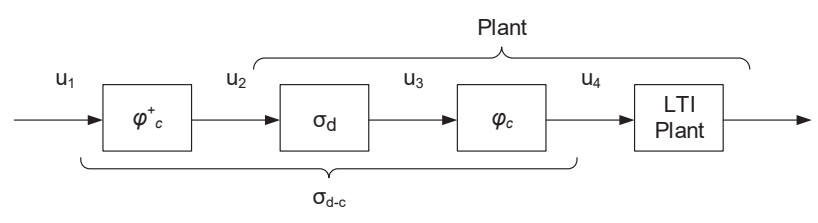

Figure 8. Backlash right inverse $\varphi_{c}^{+}$is applied to the plant input.

\subsection{Anti-windup control structure}

One can take advantage of Theorem 1 and (13) to compensate for saturation and backlash at the input of a linear plant. If an appropriate inverse to the backlash can be constructed, then the overall system can be compensated using standard anti-windup techniques. However care must be taken to set the saturation compensation at the appropriate level.

The compensation scheme proposed in this paper is illustrated in Figure 9. The plant has saturation and backlash at the input so its dynamics are given as

$$
y=\left(G \varphi_{c} \sigma_{d}\right) u_{2},
$$

where $G$ represents the linear dynamics and $u_{2}$ is the signal delivered to the plant. If $u_{2}$ is given as

$$
u_{2}=\varphi_{c}^{+} u_{1}
$$

for some $u_{1}$ then by Theorem 1

$$
u_{4}=\sigma_{d-c} u_{1}
$$

Hence the proposal is to generate $u_{1}$ using a standard linear compensator together with anti-windup set at level $d-c$. The overall control consists of a right backlash inverse and a linear compensator with anti-windup.

\subsection{Optimality of compensation}

In the proposed control scheme the input delivered to the plant $u_{2}$ is generated using a backlash inverse $u_{2}=\varphi_{c}^{+} u_{1}$, even though the plant itself has both saturation and backlash at its input. The linear controller and anti-windup can then be designed arbitrarily based on the linear plant dynamics $G$ and the saturation level $d-c$. In Section 4 this is illustrated with PID control and simple anti-windup. Nevertheless the structure is optimal in the sense that it ensures the best possible match between $u_{1}$ and $u_{4}$. Specifically

Theorem 2. Let $\varphi_{c}, \varphi_{c}^{+}$and $\sigma_{d}$ be defined as in Section 2. Suppose $u_{1} \in L_{p}[0, \infty)$ with $p \geq 1$ and let

$$
u_{4}=\left(\varphi_{c} \sigma_{d} \varphi_{c}^{+}\right) u_{1} \text { with } u_{4}(0)=\operatorname{sat}_{d-c} u_{1}(0) .
$$

Then for any causal $\psi$ such that $\left(\varphi_{c} \sigma_{d} \psi\right) u_{1} \in L_{p}[0, \infty)$,

$$
\left\|u_{1}-\left(\varphi_{c} \sigma_{d} \psi\right) u_{1}\right\|_{p} \geq\left\|u_{1}-u_{4}\right\|_{p} .
$$

Proof. Define $\tilde{u}_{3}=\left(\sigma_{d} \psi\right) u_{1}$ and $\tilde{u}_{4}=\left(\varphi_{c} \sigma_{d} \psi\right) u_{1}$. When $p<\infty$, since both $u_{1}$ and $\tilde{u}_{4}$ are Lebesgue measurable, one 


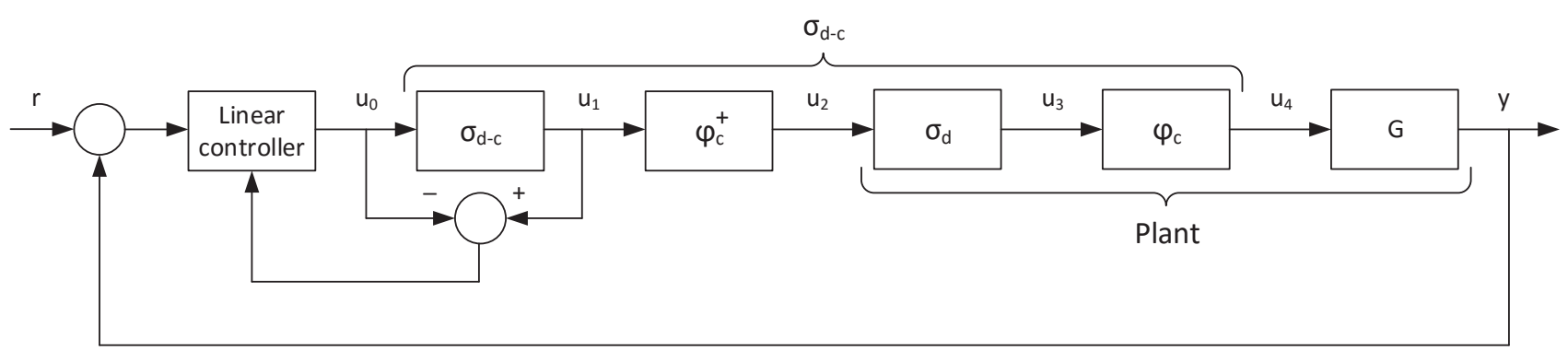

Figure 9. Proposed controller for a system subject to a nonlinearity $\varphi_{c}$ and saturation $\sigma_{d}$ in the input using a linear controller with back-calculation anti-windup and backlash right inverse. The anti-windup saturation $\sigma_{d-c}$ has a lower level than the plant saturation $\sigma_{d}$.

may write

$$
\begin{aligned}
\left(\left\|u_{1}-\tilde{u}_{4}\right\|_{p}\right)^{p} & =\int_{\mathscr{T}_{0}}\left|u_{1}(t)-\tilde{u}_{4}(t)\right|^{p} d t \\
& +\int_{\mathscr{T}_{-}}\left|u_{1}(t)-\tilde{u}_{4}(t)\right|^{p} d t \\
& +\int_{\mathscr{T}_{+}}\left|u_{1}(t)-\tilde{u}_{4}(t)\right|^{p} d t
\end{aligned}
$$

where $\mathscr{T}_{0}, \mathscr{T}_{-}$and $\mathscr{T}_{+}$are the Lebesgue measurable subsets of $[0, \infty)$ where $\left|u_{1}(t)\right| \leq d-c, u_{1}(t)<-(d-c)$ and $u_{1}(t)>$ $d-c$ respectively. Hence

$$
\begin{aligned}
\left(\left\|u_{1}-\tilde{u}_{4}\right\|_{p}\right)^{p} & \geq \int_{\mathscr{T}_{-}}\left|u_{1}(t)-\tilde{u}_{4}(t)\right|^{p} d t \\
& +\int_{\mathscr{T}_{+}}\left|u_{1}(t)-\tilde{u}_{4}(t)\right|^{p} d t .
\end{aligned}
$$

But $\left|\tilde{u}_{4}(t)\right| \leq d-c$ for all $t$ (since $\left|\tilde{u}_{3}(t)\right| \leq d$ for all $t$ ), so

$$
\begin{aligned}
\left(\left\|u_{1}-\tilde{u}_{4}\right\|_{p}\right)^{p} & \geq \int_{\mathscr{T}_{-}}\left|u_{1}(t)+d-c\right|^{p} d t \\
& +\int_{\mathscr{T}_{+}}\left|u_{1}(t)-d+c\right|^{p} d t .
\end{aligned}
$$

If $u_{4}=\left(\varphi_{c}^{+} \sigma_{d} \varphi_{c}\right) u_{1}$ and $u_{4}(0)=\operatorname{sat}_{d-c} u_{1}(0)$ then by Theorem $1, u_{4}(t)=\operatorname{sat}_{d-c} u_{1}(t)$ for all $t$. It follows that if we $u_{4}$ is substituted for $\tilde{u}_{4}$ then (21) holds with equality.
Hence

$$
\left\|u_{1}-\tilde{u}_{4}\right\|_{p} \geq\left\|u_{1}-u_{4}\right\|_{p}, \text { for any } \tilde{u}_{4}=\left(\varphi_{c} \sigma_{d} \psi\right) u_{1}
$$

A similar result also follows immediately if we let $p \rightarrow \infty$.

Theorem 2 lays the optimality of the construction introduced by Theorem 1 and proposed in Section 3.2. To appreciate this, consider Figure 7 and let $u_{4}=\left(\varphi_{c} \sigma_{d} \varphi_{c}^{+}\right) u_{1}$. Theorem 2 refers to the relationship between $u_{1}$ and $u_{4}$. In general, one would wish the designed controller to make $u_{1}=u_{4}$ (that is, to 'remove' the nonlinearities of the plant). However, as Theorem 2 shows, when a saturation and a nonlinearity, such as backlash, are present, this may not be possible. The best approximation of $u_{4}$ to $u_{1}$ is a saturation function $\sigma_{d-c}$ (i.e., $\left.u_{4}=\sigma_{d-c} \mathrm{u}_{1}\right)$. Notice that the saturation $\sigma_{d-c}$ is more restrictive than the original plant saturation $\sigma_{d}$. 


\section{Experimental Results}

The results are demonstrated in an HP Deskjet 3000 printer, both in simulation and experimentally. This control kit was developed by [20] at The University of Manchester. The objective of the experiment is to control the position of the printer head, which is driven by a DC motor. The head position is sent to an ELVIS Power Interface Daughter Board through a digital encoder. The Daughter Board sends this signal to the PC using an ELVIS Board. In the PC, the control signal is calculated using LabVIEW, and then sent back to the printer via the ELVIS Board. The experimental setup is shown in Figure 10.

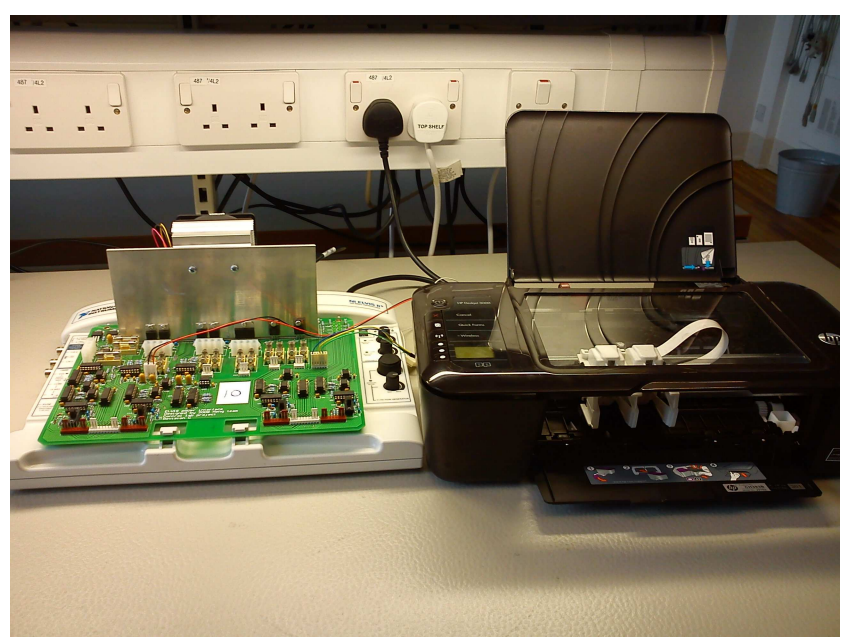

Figure 10. Printer and ELVIS and ELVIS Power Interface

Daughter Boards.

The identification of the printer's model yields the following model

$$
\hat{G}(s)=\frac{1144.9}{s(s+78.6)}
$$

The system's structure is that shown in Figure 9. Simple PID suffices for the linear controller since the printer model is second order.

\subsection{Simulation results}

The simulations are run for backlash with saturation to correspond with the experimental case. Three simulations are performed using different variations of the controller, namely:

\section{Scheme 1}

(a) PID (saturation active).

(b) PID with anti-windup (saturation active).

\section{Scheme 2}

(a) Saturation equivalence with PID with antiwindup (saturation active).

(b) Saturation equivalence with PID with antiwindup (saturation active) and anti-chattering.

Schemes 1(a) and 1(b), do not include a specific compensation for the nonlinearity. They are constructed to compare their response against the proposed controller (saturation equivalence).

The gains of the PID are chosen as $K_{p}=2.5, K_{i}=\frac{K_{p}}{T_{i}}=5$, $K_{d}=K_{p} T_{d}=0.00025 \approx 0$, with the gain in the anti-windup feedback set as $T_{a}=T_{i}$. The anti-windup augmentation compensates for the equivalent saturation sat ${ }_{d-c}$, like in 
Figure 9. The parameter that describes backlash is set to $c=0.75$ and the saturation level is assumed to be at $d=3$.

Remark: In the simulations and experiments, the reference $r(t)$ and the output $y(t)$ represent the desired and actual position of the printer head. They are scaled from 0 to 5 , where 5 corresponds to the end of the rail. The rail itself is $38 \mathrm{~cm}$, while the printer head measures $8 \mathrm{~cm}$, which means that there is $30 \mathrm{~cm}$ movement end-to-end.

Scheme 1(a): The output of the input saturated system using a standard PID is shown in the top part of Figure 11. In this case, the output not only presents an overshoot caused by the saturation in the actuator which winds the integral term of the PID up, it can also be observed that the response oscillates around the setpoint due to the presence of backlash. The control signal in this case appears at the bottom of Figure 11.

Scheme 1(b): Now, the input saturated plant is controlled using a PID with anti-windup. As Figure 12 illustrates, the anti-windup augmentation reduces the overshoot in the response, but it does nothing to compensate for the backlash nonlinearity. The control signal in this case appears at the bottom of the same figure.

Scheme 2(a): Next, the proposed controller, using the inverse backlash and the PID with anti-windup, adjusted to the saturation equivalence $\sigma_{d-c}$ is built. In this case, the oscillations caused by the backlash nonlinearity are eliminated, obtaining a nice tracking of the reference signal.
This is presented in Figure 13 (top). The control signal $u$, which corresponds to $u_{2}$ in Figure 9, is plotted at the bottom of the same figure.

Scheme 2(b): It can be observed from Figure 13, that the signal $u$ shows chattering. The backlash inverse presents discontinuities everywhere, which means that the chattering can occur anywhere. However, the chattering can be avoided, as in the case of deadzone, using an anti-chattering solution which can be classified as spatial regularization, see [17]. The algorithm for such regulator is given in Algorithm 1. In the algorithm, uap is a variable indicating the sign of $\dot{u}_{1}$, and $u_{s}$ indicates at which side of the backlash inverse the output should be located, depending on the sign of uap.

Algorithm 1: Anti-chattering regulator for backlash

inverse.

Data: uap,usp,c,error

Result: us

if $a b s$ (error) $\geq 0.00001$ then

if $u a p<0$ then $u s=-c$;

if $u a p>0$ then $u s=c$;

else $u s=u s p$;

else

$u s=u s p$

end 
Figure 14 (bottom) presents the control signal $u$ when same figure it is clear that the anti-chattering compensator using an anti-chattering solution. From the top plot of the
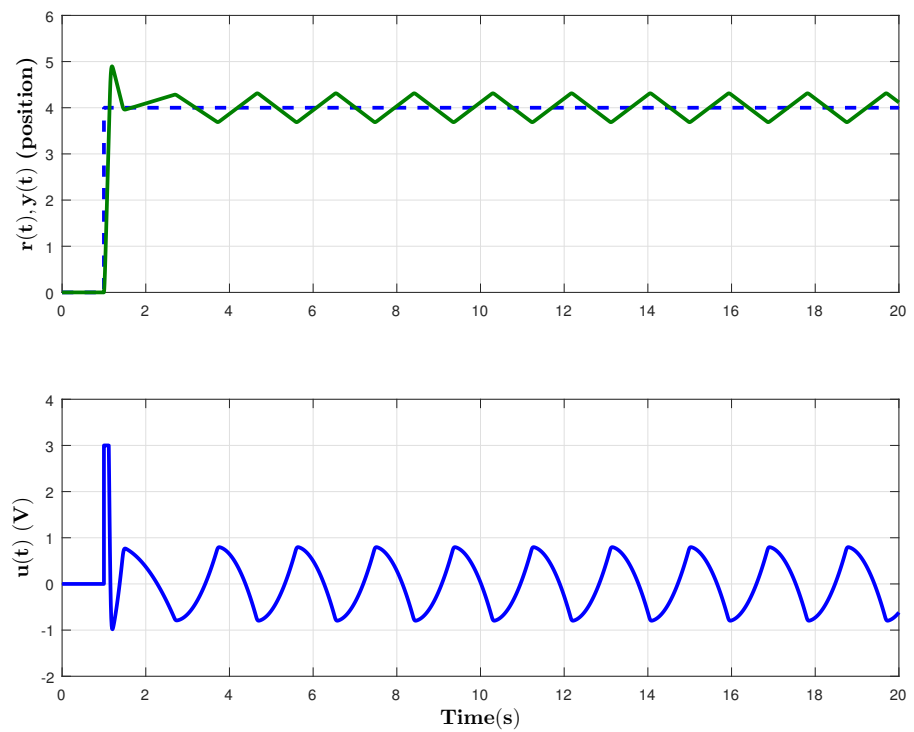

Figure 11. Scheme 1(a). System response for a plant with saturation and backlash in the input, using a PID controller (top) and the corresponding control signal $u$ representing $u_{2}$ in Figure 9 (bottom).
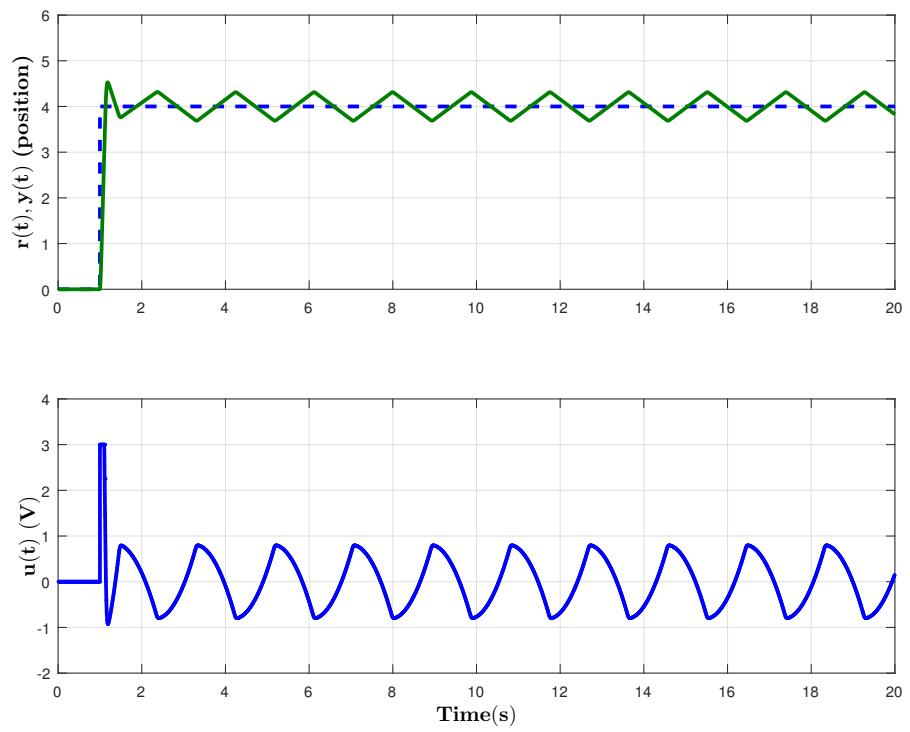

Figure 12. Scheme 1(b): System response for a plant with saturation and backlash in the input using a PID controller with anti-windup augmentation (top), and control signal $u$ (which corresponds to $u_{2}$ in Figure 9 (bottom). 
described in Algorithm 1 does not affect the response of the system.

The example above illustrates the applicability of Theorem 1. In this case, anti-windup is used to exploit the implications of the theorem, though other more specific anti-windup compensators can be used, as described by Tarbouriech et al. in[11] and by Zaccarian and Teel in [8].

\subsection{Laboratory results}

The results in this section correspond to the experiments carried out on the printer shown in Figure 10 using an anti-windup controller. The plant is first run in openloop to identify the type of nonlinearity it is subject to. From this first experiment it is concluded that the affecting nonlinearity is a backlash with deadband of size $2 c=1.5$, as can be appreciated in Figure 15. Moreover, the control signal is saturated by a limit in the DC motor voltage of $\pm 3 V$. Then, from Theorem 1 , the nonlinearities acting on the plant correspond to a saturation given by $d-c=2.25$.

A set of experiments were performed, first using a standard PID controller with anti-windup, as in Figure 3 and then using the results from section 3. as in Figure 9.

For each controller a reference signal $r(t)=A \sin (\omega t)$, with $A=1$ and $\omega=2 \pi \mathrm{rad} / \mathrm{s}$ was assumed. The PID gains in both cases were set as $K_{p}=2.5, K_{i}=5$ and $K_{d}=0.00001$. Note that the derivative actions is practically zero.
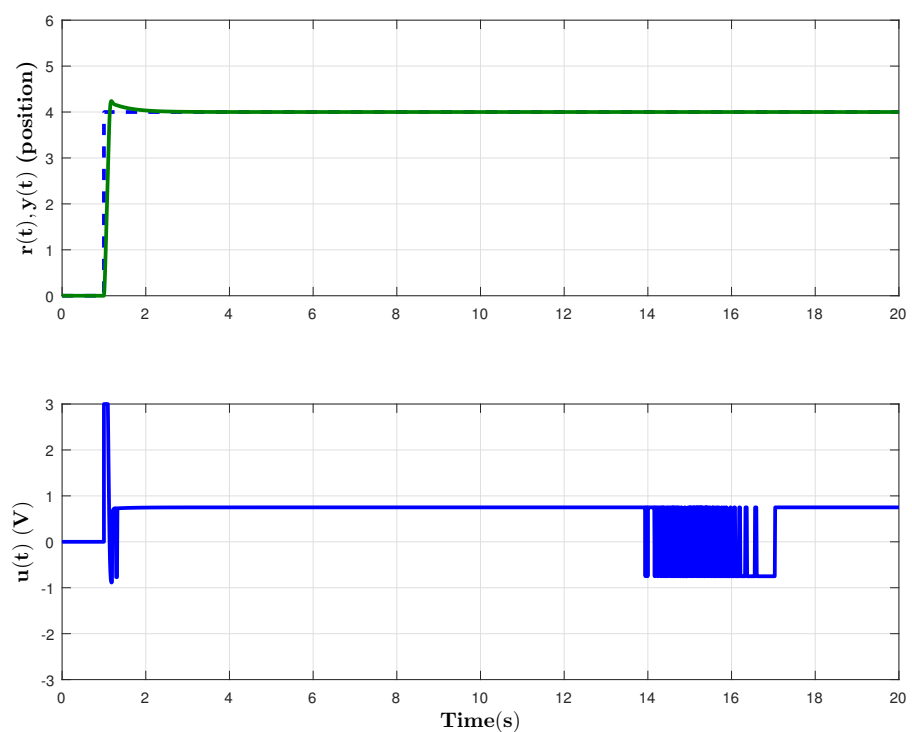

Figure 13. Scheme 2 (a): System response for a plant with saturation and backlash in the input using the proposed saturation equivalence, combined with a PID with antiwindup controller (top), and the corresponding control signal (bottom). Note that chattering in the control signal $u$, induced by the inverse backlash. 

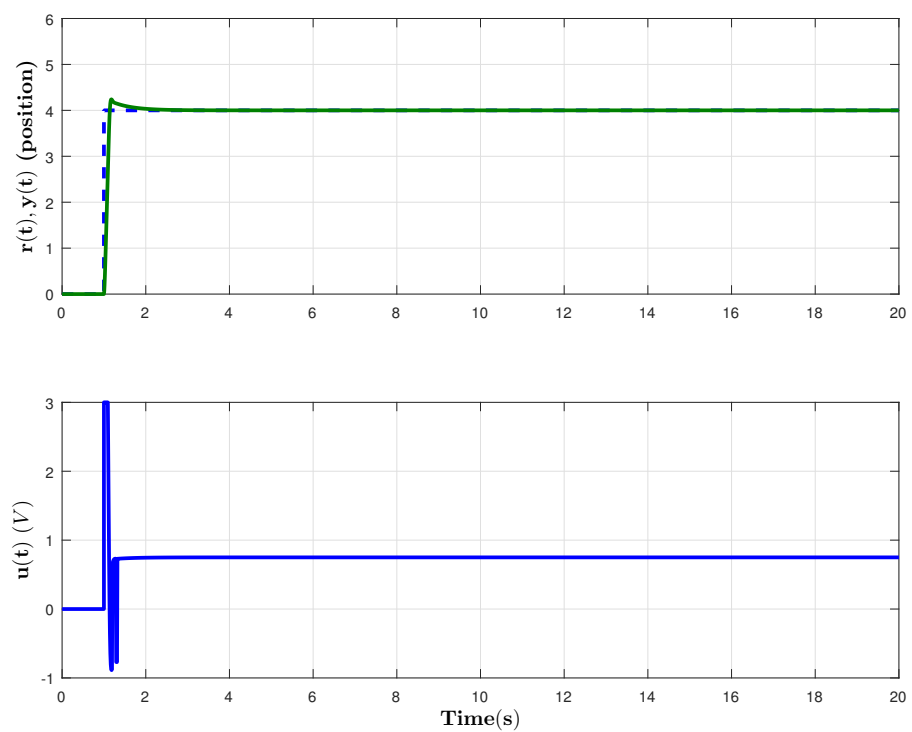

Figure 14. Scheme 2 (b):System response for a plant with saturation and backlash in the input using the proposed 'saturation equivalence' controller with anti-chattering regulation (top), and the corresponding control signal (bottom).

The response obtained with the standard PID and compared to the standard PID controller. The presence of the proposed controller are shown in figs. 16 and 17, the backlash nonlinearity could call for a more complex respectively. From the figures it is easy to see that the control strategy, however the results of Theorem 1 allow inverse backlash compensator offers a better response us to use a simple anti-windup scheme that offers improved results.

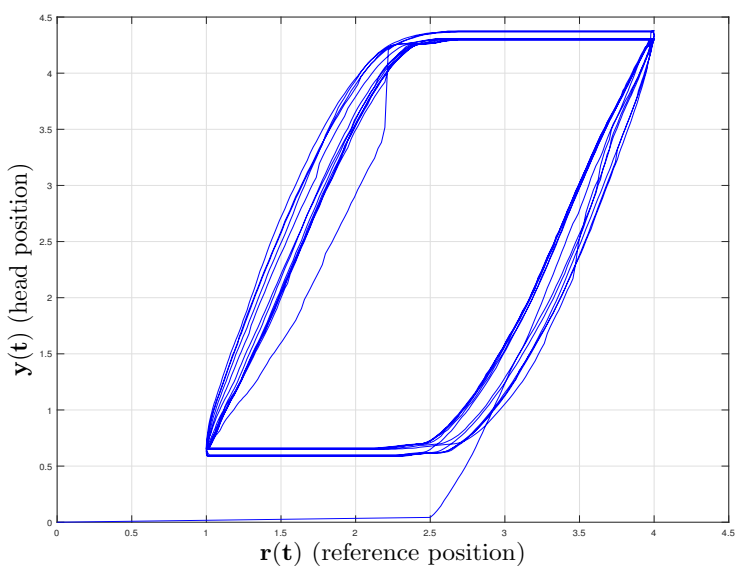

The step response of the closed-loop system using the standard PID with anti-windup is shown at the top part of Figure 18. The control signal in this case is presented at the bottom of the same figure.

Similarly, Figure 19 shows the response to the step using the proposed saturation equivalence controller, combined with a PID with anti-windup. The proposed controller provides better results by removing the oscillations that are

Figure 15. Input versus output plot of the printer in open-loop. evident in the standard PID plus anti-windup case. Note that 


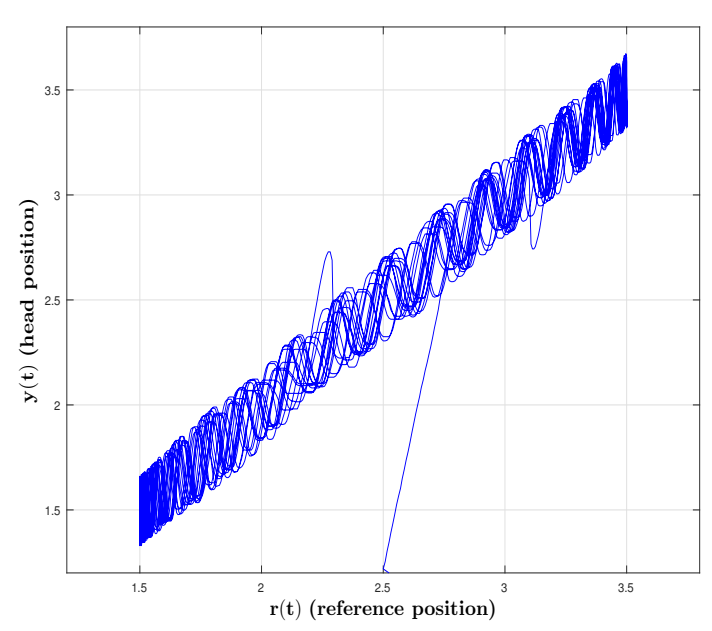

Figure 16. Input versus output plot of the printer in closed-loop using a standard PID with anti-windup compensation.

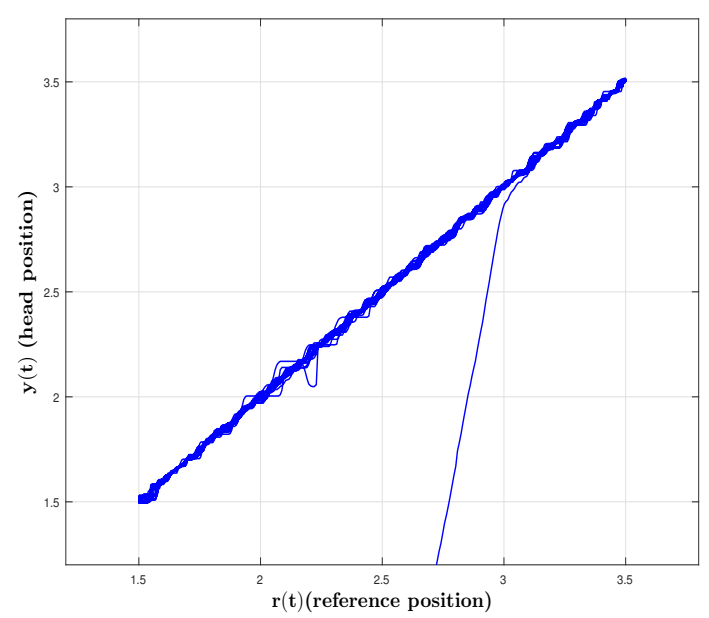

Figure 17. Input versus output plot of the printer in closed-loop using the proposed 'saturation equivalence' controller with anti-windup compensation.

when using only the PID with anti-windup the controller struggles to keep the printer head at the reference, while the proposed controller reaches the steady state effectively.
However, it is observed that when using the proposed controller the control signal $u_{2}$ presents chattering in steady-state, as shown in the bottom part of Figure 19.

As in the simulations, a spatial regularization was added to the backlash inverse to remove such chattering. The effects on the control signal $u$ (i.e. $u_{2}$ in Figure 9) and the step response can be observed in Figure 20 (bottom and top plots, respectively). Notice that the anti-chattering does not negatively affect the response of the system compared with the case when chattering is present, see Figure 19

\section{Conclusions}

This paper is dedicated to the control of plants that exhibit saturation and backlash in the input. A result is obtained that shows that the linear interconnection of an inverse nonlinearity, a saturation, and a nonlinearity (backlash), is equivalent to a saturation whose parameters depend on the parameters of the original nonlinearities. It is shown, as well, that this solution is optimal in the sense that when saturation and backlash are present in a plant's input, the best one can expect is to compensate for a saturation $\sigma_{d-c}$, which is achieved by building an inverse for the affecting nonlinearity. The results are later demonstrated in simulation and experimental using a basic anti-windup controller to compensate for the obtained saturation. 

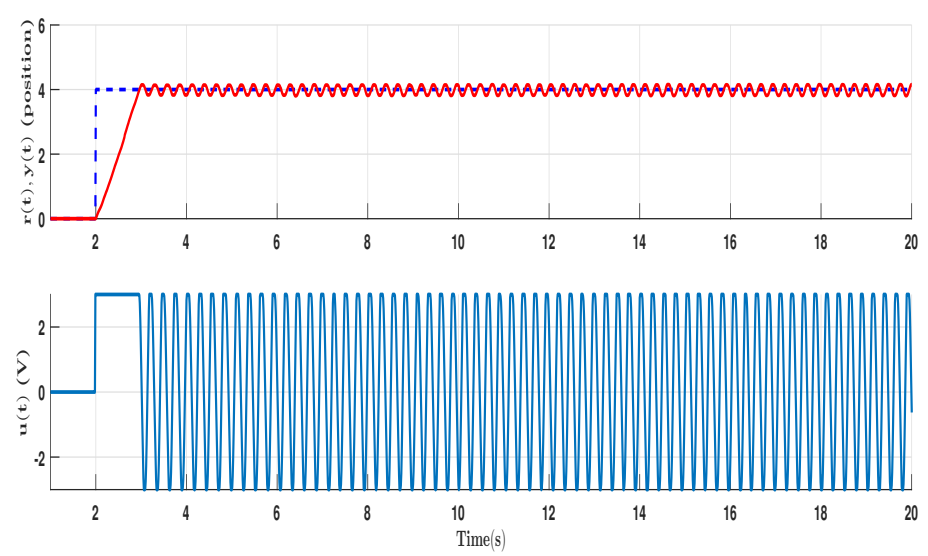

Figure 18. Step response of the plant using a standard PID with anti-windup control (top). Control signal $u$ applied to the plant (bottom).
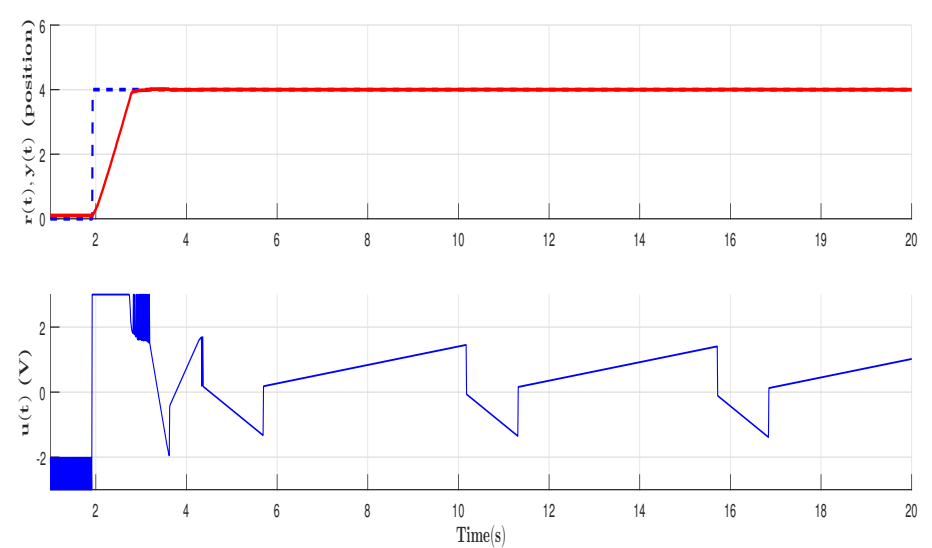

Figure 19. Step response of the system in closed-loop using the proposed saturation equivalence controller with a PID with anti-windup (top). Control signal $u$ when using the saturation equivalence controller with anti-windup compensation (bottom). Note that the signal is affected by chattering in steady state.

\section{Acknowledgements}

Authors would like to thank CONACyT for the funding provided for the realization of this work.

\section{Notes}

1. Some of the work presented in this paper was done while the first author was at The University of Manchester, United
Kingdom, and some while at Universidad Autónoma de San

Luis Potosí, Mexico.

\section{References}

[1] Tao G and Kokotović P. Adaptive control of systems with actuator and sensor nonlinearities. John Wiley and Sons, 1996. 

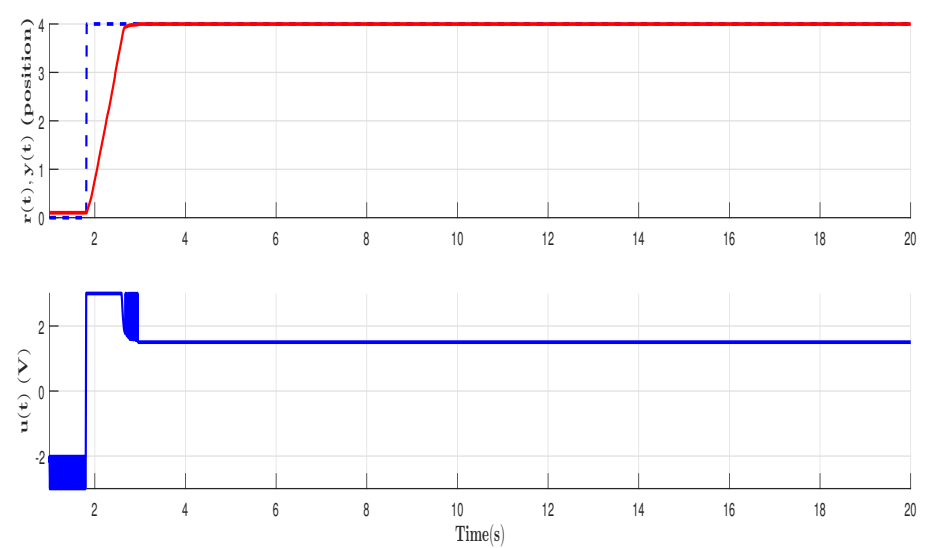

Figure 20. Step response of the system in closed-loop using the saturation equivalence controller with an anti-chattering strategy (top). Control signal $u$ when using the proposed controller with anti-windup and an anti-chattering strategy (bottom).

[2] Zhou J and Wen C. Inverse control of systems with backlash nonlinearity. In Adaptive Backstepping Control of Uncertain Systems, Lecture Notes in Control and Information Sciences, volume 372. Springer Berlin Heidelberg, 2008. pp. 125-138.

[3] Agrawal V, Peine W, Yao B et al. Control of cable actuated devices using smooth backlash inverse. In 2010 IEEE Int Conf Robot. pp. 1074-1079.

[4] Selmic R and Lewis F. Backlash compensation in nonlinear systems using dynamic inversion by neural networks. In 1999 IEEE Int Conf Contr. pp. 11631168.

[5] Barbosa R, Teneiro J and Galhano A. Performance of fractional PID algorithms controlling nonlinear systems with saturation and backlash phenomena. $J$ Vib Control 2007; 13(9-10): 1407-1418.
[6] Nordin M and Gutman PO. Controlling mechanical systems with backlash - a survey. Automatica 2002; 38: 1633-1649.

[7] Galeani S, Tarbouriech S, Turner MC et al. A tutorial on modern anti-windup design. Eur J Control 2009; 15(3-4): 418-440.

[8] Zaccarian L and Teel AR. Modern Anti-windup Synthesis. Princeton, 2011.

[9] Åstrom K and Hägglund T. PID controllers: theory, design and tuning. Instrument Society of America, 1995.

[10] Glattfelder $\mathrm{AH}$ and Schaufelberger W. Control systems with input and output constraints. SpringerVerlag London Limited, 2003. 
[11] Tarbouriech S and Turner M. Anti-windup design: an overview of some recent advances and open problems. IET Control Theory A 2007; 3(1): 1-19.

[12] Zabiri H and Samyudia Y. MPC design for contrained multivariable systems under actuator backlash. pp. 643-648.

[13] Zabiri H and Samyudia Y. A hybrid formulation and design of model predictive control for systems under actuator saturation and backlash. J Process Contr 2006; 16: 693-709.

[14] Tarbouriech S, Prieur C, Queinnec I et al. Global stability for systems with nested backlash and saturation operators. In 2010 Amer Contr Conf. pp. 2665-2670.

[15] Tarbouriech S, Queinnec I, Alamo T et al. Ultimate bounded stability and stabilization of linear systems interconnected with generalized saturated functions. Automatica 2011; 47: 1473-1481.

[16] Tarbouriech S, Queinnec I and Prieur C. Stability analysis for systems with saturation and backlash in the loop. In 52nd IEEE CDC. pp. 6652-6657.

[17] Johansson KH, Egerstedt M, Lygeros J et al. On the regularization of hybrid automata. Syst Contr Lett 1999; 38: 141-150.
[18] Rodríguez-Liñán MC and Heath WP. Controller structure for plants with combined saturation and deadzone/backlash. In 2012 IEEE International Conference on Control Applications (CCA) Part of 2012 IEEE Multi-Conference on Systems and Control. pp. 1394-1399.

[19] Rodríguez-Liñán MC and Heath WP. MPC for plants subject to saturation and deadzone, backlash or stiction. In IFAC Conference NMPC'12, volume 4 Pt. 1. pp. $418-423$.

[20] Heath WP, Onel PM Oand Green, Lennox B et al. Developing a student-focused undergraduate laboratory. Int J Elec Eng Educ 2013; 50(3). 